Research Article

\title{
Performance of 2D Compressive Sensing on Wide-Beam Through-the-Wall Imaging
}

\author{
Edison Cristofani, Mathias Becquaert, and Marijke Vandewal \\ CISS Department, Royal Military Academy, 30 Avenue de la Renaissance, 1000 Brussels, Belgium \\ Correspondence should be addressed to Edison Cristofani; edison.cristofani@elec.rma.ac.be
}

Received 27 May 2013; Revised 18 July 2013; Accepted 24 July 2013

Academic Editor: Sandra Costanzo

Copyright ( 2013 Edison Cristofani et al. This is an open access article distributed under the Creative Commons Attribution License, which permits unrestricted use, distribution, and reproduction in any medium, provided the original work is properly cited.

Compressive sensing has become an accepted and powerful alternative to conventional data sampling schemes. Hardware simplicity, data, and measurement time reduction and simplified imagery are some of its most attractive strengths. This work aims at exploring the possibilities of using sparse vector recovery theory for actual engineering and defense- and security-oriented applications. Conventional through-the-wall imaging using a synthetic aperture configuration can also take advantage of compressive sensing by reducing data acquisition rates and omitting certain azimuth scanning positions. An ultra-wideband stepped frequency system carrying wide beam antennas performs through-the-wall imaging of a real scene, including a hollow concrete block wall and a corner reflector behind it. Random downsampling rates lower than those announced by Nyquist's theorem both in the fast-time and azimuth domains are studied, as well as downsampling limitations for accurate imaging. Separate dictionaries are considered and modeled depending on the objects to be reconstructed: walls or point targets. Results show that an easy interpretation of through-the-wall scenes using the $l_{1}$-norm and orthogonal matching pursuit algorithms is possible thanks to the simplification of the reconstructed scene, for which only as low as $25 \%$ of the conventional SAR data are needed.

\section{Introduction}

Previous research studies have explored numerous applications of through-the-wall imaging (TWI) in domains which can exploit the convenient penetration capabilities of the 1$5 \mathrm{GHz}$ frequency band in most nonmetal building construction materials $[1,2]$. Indeed, being able to perform nondestructive inspection of objects behind a solid wall is a noteworthy application. The literature on TWI widely copes with object or human detection-either moving [3-5] or static $[6,7]$. Among others, military, law enforcement, and security operations have already taken profit of the satisfactory performance of TWI. Although most of the studies suggest using an active TWI approach wherever needed, some authors opt for adequately processing opportunistic signals in the surroundings of the scene such as widespread GSM [8] or wireless networks [9]. Despite presenting interesting wall penetration properties, the noncooperative-thus passive-nature of these approaches cannot assure complete reliability and availability, and consequently, an active approach is preferred.
Synthetic Aperture Radar (SAR) allows competitive TWI using rather common antennas with wide beams and simple out-of-focus data acquisition. Thanks to coherent signal integration along a known measurement path, these data are focused on postprocessing, yielding an improved lateral resolution which remains constant at all ranges [10]. Given a measurement path, sufficient data both in the azimuth and fast-time directions must be obtained in order to perform SAR focusing and to satisfy the Nyquist-Shannon antialiasing criterion. This, generally, implies using complex hardware which generates amounts of data which can become unbearable when fine resolution imagery is needed.

Compressive Sensing (CS) introduces the possibility of reducing the amount of sensed signals for further digital reconstruction and, likewise, to perform accurate TWI. First used to solve sparse vector recovery problems within mathematical applications, CS has been recently used in a growing list of fields for sparse signal reconstruction, including TWI applications [11-14]. CS is able to reconstruct signals with high probability provided random sampling and sufficient 
sparsity levels are satisfied [15]. Since CS announces a convex function optimization problem, solutions can be found by using the $l_{1}$-norm minimization or, in a less demanding approach, the Orthogonal Matching Pursuit (OMP) greedy algorithm, among an endless list of possibilities.

Actual TWI experiments were conducted for this study. Hollow concrete stretcher blocks were used to build up a solid wall, replicating load-bearing foundation walls used in most permanent constructions. A corner reflector, serving as an object of interest, was placed behind the wall. Scene reconstruction using an ultra-wideband moving sensor was achieved, both in conventional SAR and CS approaches. This study will explore the possibilities of random sampling, with a focus on minimizing the amount of data required to produce accurate enough reconstructions of simple TWI scenes. This action will lead to a significant simplification in hardware, as well as reducing processing complexity and data handling. The remaining of this document is divided into four further sections: Section 2 describes the used experimental setup, as well as signal processing concepts that will be used throughout the work. Section 3 formulates the CS problem to sparse data recovery, and strategies for creating dictionaries are described. Section 4 presents conventional and CS results obtained from actual TWI measurements. Finally, Section 5 details conclusions drawn and future work.

\section{Conventional TWI Measurements}

2.1. TWI and the Measurement Site. The sensor used for the presented TWI comprises two Schwarzbeck BBHA 9120 D horn antennas [16] (for transmitting and receiving, providing gain stability in the $1-5 \mathrm{GHz}$ band) on a stepped frequency (SF) SAR moving scanner, which translates for $1500 \mathrm{~mm}$ following an equispaced azimuth sampling scheme. The sensor used is an Anritsu 37169A Vector Network Analyzer (VNA). A concrete wall was built up for this study in order to obtain results comparable to those of a real TWI situation (see Figure 1). The wall was located at $950 \mathrm{~mm}$ from the antennas, in the far-field region for the entire sensor's frequency band, and a corner reflector was placed at $1240 \mathrm{~mm}$ behind the wall.

Hollow concrete stretcher blocks were used (see Figure 2 for shape and dimensions), commonly found in masonry and especially in load-bearing foundation walls.

Range estimation is greatly affected by the change of relative permittivity occurring in the air-material interfaces. Consequently, imaged targets and walls will appear further away and wider than in reality. Given a material with relative permittivity $\epsilon_{r}>1$, any transmitted electromagnetic wave through it will propagate at a speed $v=c / \sqrt{\epsilon_{r}}$, being $c$ the speed of light in vacuum. For an air-concrete-air interface of $35 \mathrm{~mm}$, and considering $\epsilon_{r} \sim 4$ approximately [17], the second interface would be located at $d=+70 \mathrm{~mm}$, or $\Delta d=$ $+35 \mathrm{~mm}$ further away. As depicted in Figure 3, any object behind a concrete wall will suffer from this phenomenon, whereas its actual location is closer than shown by TWI. Waves traveling through hollow concrete blocks, as those used in the proposed experiments, will encounter two 35-mm

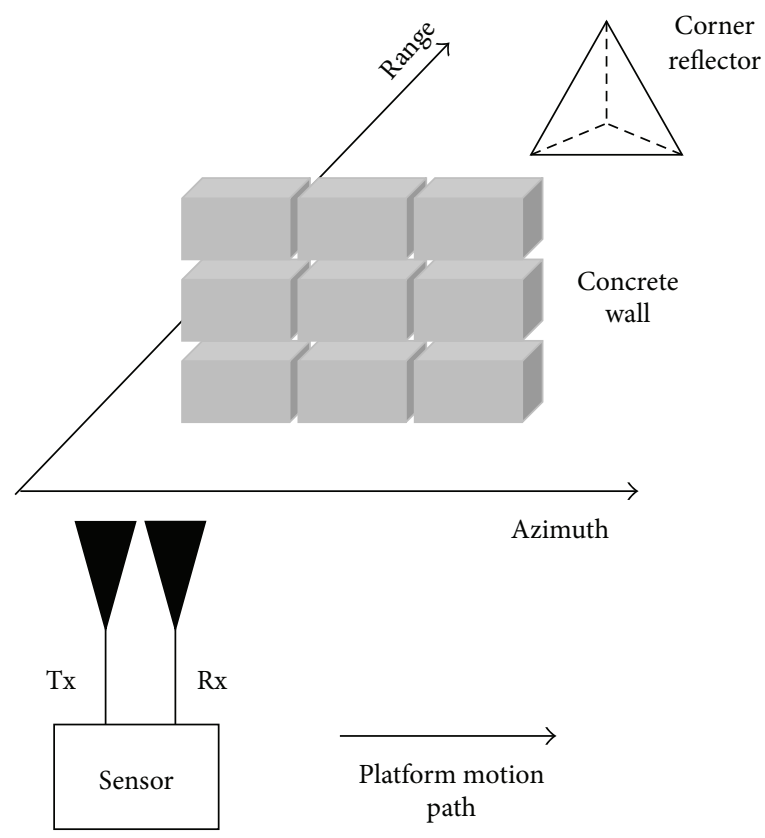

FIGURE 1: Schematic representation of the proposed experimental setup.

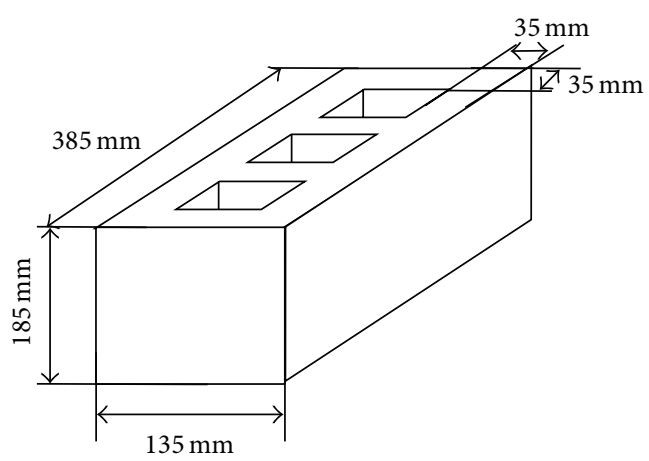

Figure 2: Dimensions of a hollow concrete stretcher block are $385 \mathrm{~mm} \times 185 \mathrm{~mm} \times 135 \mathrm{~mm}$ (length, height, and width, resp.). The outer structure is $35 \mathrm{~mm}$ thick.

air-concrete-air transitions, and therefore the expected range of the corner reflector would be shifted to ca. $2 \Delta d=+70 \mathrm{~mm}$.

2.2. The SF Sensor and Signal Model. The SAR scanner operated in this study is based on an SF ultra-wideband sensor in the $1-5 \mathrm{GHz}$ band. Using such SF sensor guarantees low hardware requirements, since the instantaneous bandwidth is kept narrow, while achieving a remarkable range resolution and power output. Instead of transmitting a linear frequency modulated chirp using a continuous or pulsed approach, the SF sensor produces signals by sequentially generating $N$ carrier frequencies covering an effective system bandwidth $B$. These frequencies pulses are described by

$$
f_{n}=f_{0}+n \Delta f \quad \text { being } n=1, \ldots, N-1,
$$

where $f_{n}$ is the $n$th frequency generated, $f_{0}$ is the starting frequency (i.e., $1 \mathrm{GHz}$ for this setup), and $\Delta f=B /(N-1)$ 


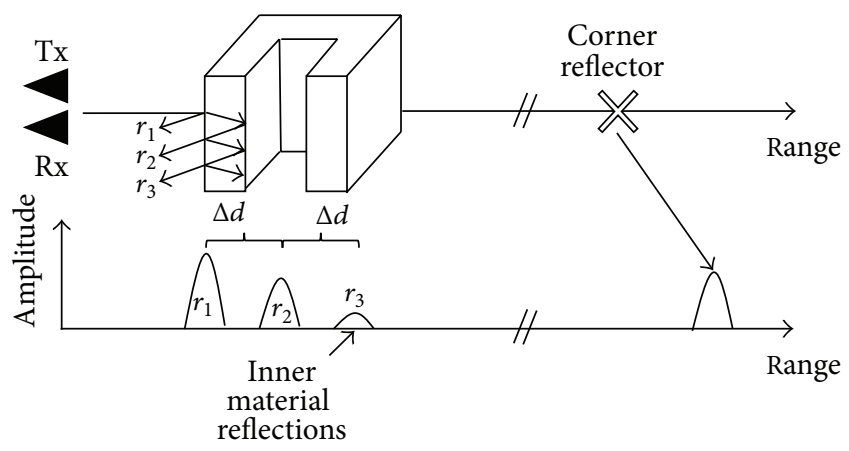

FIGURE 3: The ranging information is altered due to the different transmission media in the wall. Objects will appear further away.

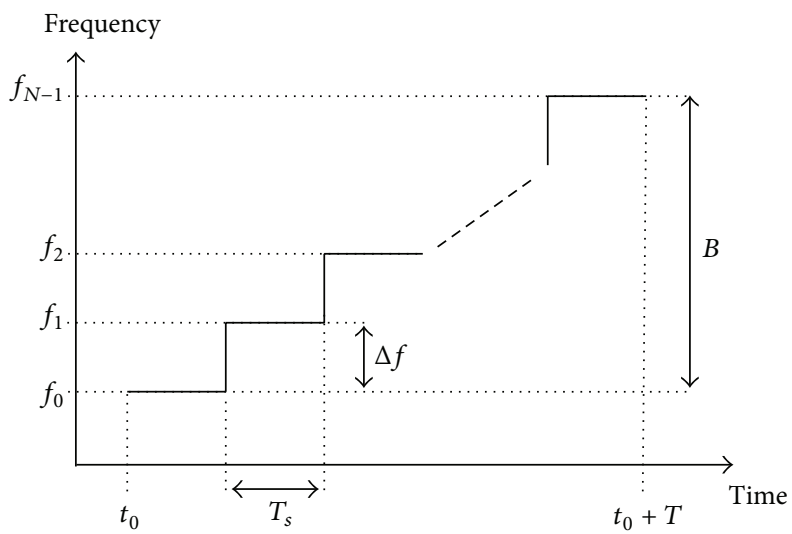

FIGURE 4: Waveform generation scheme of an SF sensor.

is the frequency step. Figure 4 depicts how the transmitted signals are generated.

For any SF systems, the maximum unambiguous range and range resolution parameters are respectively obtained as follows:

$$
\begin{gathered}
R_{\max }=\frac{c}{2 \Delta f}, \\
\Delta r=\frac{c}{2(N-1) \Delta f}=\frac{c}{2 B} .
\end{gathered}
$$

In Table 1, the most important parameters of the available experimental setup are shown. During the waveform generation phase, each frequency pulse $f_{n}$ is transmitted for a given time $T_{s}$. Averaging is performed in detection in order to reduce noise and spurious contributions to the signal of interest.

The signal model of the transmitted waveform $s_{t}$ for a given $f_{n}$ can be described as

$$
s_{t}\left(f_{n}, t\right) \propto \exp \left(j 2 \pi f_{n} t\right)
$$

where $t$ corresponds to the fast-time dimension. The sensor will actually transmit a series of $N$ individual frequencies
TABLE 1: Descriptive parameters of the TWI experiment.

\begin{tabular}{lc}
\hline VNA model & Anritsu 37169A \\
Start frequency & $1 \mathrm{GHz}$ \\
End frequency & $5 \mathrm{GHz}$ \\
$N$ & 101 \\
Averaging & 10 \\
Antenna opening angle & $14^{\circ}$ \\
$R_{\max }$ & $3750 \mathrm{~mm}$ \\
$\Delta f$ & $40 \mathrm{MHz}$ \\
$B$ & $4 \mathrm{GHz}$ \\
Tx power & $+10 \mathrm{dBm}$ \\
Tx/RX antennas & $11 \mathrm{dBi}$ \\
Typ. ant. gain & $1500 \mathrm{~mm} \times 2500 \mathrm{~mm}$ \\
Scene size & $37.5 \mathrm{~mm}$ \\
$\Delta r$ &
\end{tabular}

which will cover the whole bandwidth. The detected signal model can be described as follows:

$$
s_{d}(t) \propto \sum_{k=0}^{K-1} \sum_{n=0}^{N-1} \exp \left(j 2 \pi f_{n}\left(t-\tau_{k}\right)\right),
$$

where $\tau_{k}=2 r_{k} / c$ corresponds to the delay caused by the round-trip propagation time of a given $k$ th scatterer in the scene located at a distance $r_{k}$ from the sensor. Distance $r_{k}$ is simply obtained as the sensor-to-scatterer Euclidean distance. The VNA performs a homogeneous demodulation of the detected signal, which is known as beat signal and is modeled as

$$
s_{b}(t)=s(t) \cdot \exp \left(-j 2 \pi f_{n} t\right) \propto \sum_{k=0}^{K-1} \sum_{n=0}^{N-1} \exp \left(-j 2 \pi f_{n} \tau_{k}\right) .
$$

2.3. SAR Imaging. SAR measurements use relatively simple antennas with a small aperture length and, therefore, poor azimuth resolution. By translating the sensor as shown in Figure 5 appropriately and coherently combining received signals from the different azimuth positions, the equivalent physical length of the aperture can be increased. An antenna of a theoretically unlimited aperture length could be synthesized yielding very fine azimuth resolutions. Building such antenna would not be feasible in practice; therefore, SAR processing results in a useful tool.

Collected unfocused SAR data must be combined coherently using an SAR focusing algorithm. These algorithms perform range/azimuth compression in order to compact received energy, whether in the time or frequency domains, and are mostly based on the matched filter [18-20] assuming the prior knowledge of the transmitted signal model. Figure 6 shows a simplified representation of a range-compressed SAR measurement based on the experimental setup described in Section 2.1.

The distance between the sensor's positions along the azimuth measurement track and any point in the scene is computed using the Euclidean distance and will follow a hyperbolic function. The collected energy from any given 


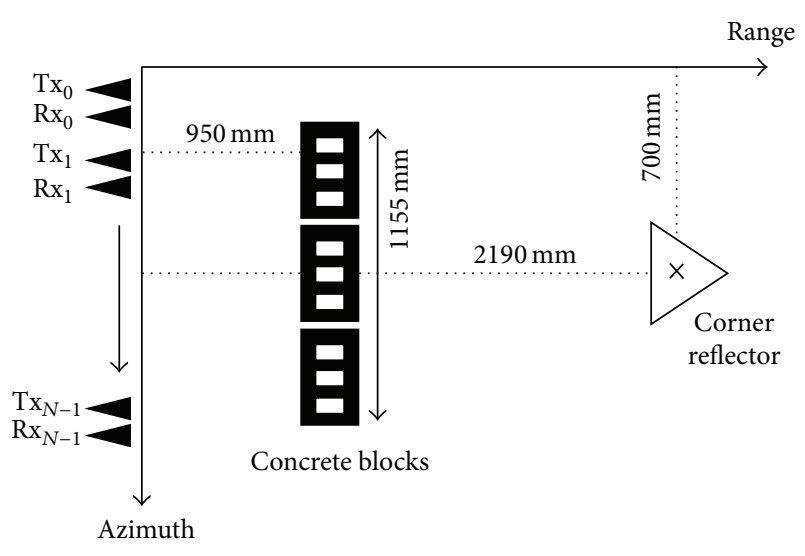

FIgURE 5: Experimental scene description. The SAR sensor moves in azimuth illuminating the scene with a wide-beam antenna.

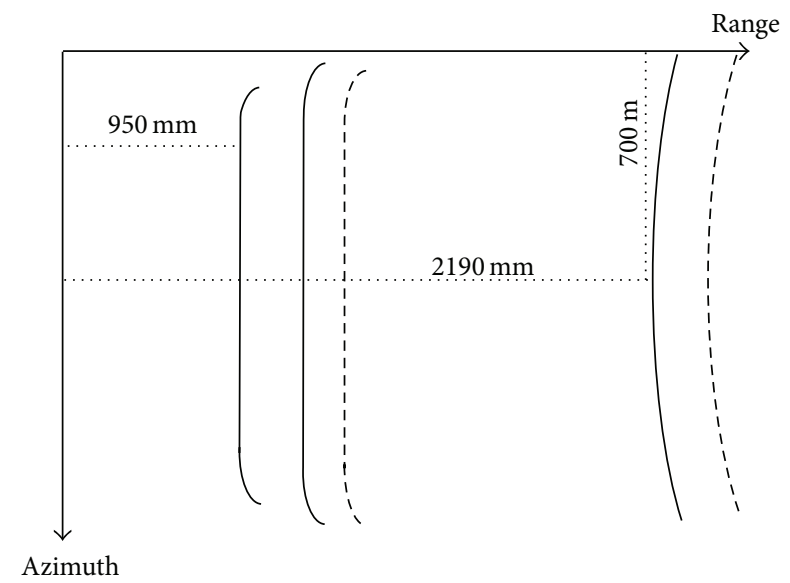

FIGURE 6: Expected results after an SAR measurement. Energy is spread in azimuth following a hyperbolic function. Certain elements will be shifted in range (dashed lines).

point target will present such hyperbolic shape and an amplitude variation in function of the antenna radiation diagram (both transmitting and receiving antennas).

Produced SAR images achieve interesting improved azimuth resolutions, which can be theoretically computed as

$$
\delta_{a z} \cong \frac{c}{2 f_{c} \sin \left(\theta_{A}\right)}
$$

where $f_{c}$ is the carrier or center frequency of the sensor and $\theta_{A}$ is the opening angle of the antenna. According to the used sensor described in Table 1, expected resolutions range and azimuth are $\delta_{r}=37.5 \mathrm{~mm}$ and $\delta_{a z}=207 \mathrm{~mm}$, respectively.

\section{Compressive Sensing}

3.1. Conventional Sensing. In signal acquisition and processing, the Nyquist-Shannon theorem [21] specifies that any signal can be perfectly reconstructed only after being uniformly sampled at, at least, twice the maximum frequency or bandwidth describing it. Consider

$$
f_{s} \geq 2 f_{\max }=2 B,
$$

where $f_{s}$ and $f_{\max }$ are the sampling and maximum frequencies, respectively. This condition can be a remarkable burden for certain systems, where challenging amounts of data are needed when large areas must be measured, high bandwidth is needed, or a combination of both. Handling, storing, or transmitting large amounts of data are onerous tasks, and SAR imaging systems are affected. Complexity of analogto-digital converters would increase if fine-resolution SAR imagery was needed.

3.2. Problem Formulation. Data recovery of sparse vectors is extensively described in the literature $[15,22,23]$. Let $x \in \mathbb{C}^{N}$ be an arbitrary $N$-dimensional vector or complete measurement, and let $y \in \mathbb{C}^{P}$ be an incomplete observation of $x$, being $P \ll N$. Vectors $x$ and $y$ are related to the linear expression

$$
y=\mathbf{A} x,
$$

where $\mathbf{A} \in \mathbb{R}^{P x N}$ is a measurement matrix containing a plausible observation scheme. Illustratively, the $n$th element of $x$ would be stored in the pth element of $y$ if $a_{p n} \neq 0$. A perfect recovery of vector $x$ can be obtained from $y$ if two conditions are fulfilled: vector $x$ must be sufficiently sparse and matrix A must satisfy the Restricted Isometry Property (RIP) $[15,22]$. Vector $x$ is said to be $S$-sparse if the $l_{0}$-norm of $x$ satisfies $\|x\|_{0} \leq S$ that is, the maximum number of nonzero elements describing $x$ must be $S$. Nevertheless, having such a vector satisfying the $S$-sparsity condition or demonstrating the RIP for all the possible combinations can be cumbersome tasks, if not implausible. This latter requirement can be relaxed by defining matrix $\mathbf{A}$ following a random distribution which satisfies the RIP with high probability [24].

In radar imaging, vector $x$ is defined as the full observation of the returns from an illuminated scene. Provided that the signal model is known (refer to Section 2.2), vector $x$ can be expressed as

$$
x=\Psi \sigma,
$$

where for all $L$ positions in the scene, $\sigma \in \mathbb{C}^{L}$ describes the reflectivity coefficients, being $P \leq L \ll N$, whereas matrix $\Psi \in \mathbb{C}^{N x L}$, an over complete dictionary, contains fully sampled received signals as described by the theoretical signal model. Therefore, $\Psi$ is populated column wise as

$$
\Psi=\left[s_{0}, s_{1}, \ldots, s_{L-1}\right] .
$$

Let then $\Phi \in \mathbb{R}^{P x N}$ be a matrix containing either zeros or ones, in perfect analogy to matrix $\mathbf{A}$, describing a certain sampling scheme. Let a sensor obey such scheme; the sensed signal $y$ for a given $\sigma$ is now defined as

$$
y=\boldsymbol{\Phi} x=\boldsymbol{\Phi} \Psi \sigma=\Theta \sigma .
$$


Inputs:

(i) measured incomplete signal $y$ and matrix $\Theta$, populated with simulated wall or point-target model data

(ii) maximum number of iterations $K$

Output: reconstructed scene vector $\widehat{\sigma}$

(1) Initialize residual $r_{0}$ to the measured signal vector $y$ and secondary matrix $\Theta^{\dagger}$ to zero

$$
r_{0}=y, \quad \Theta^{\dagger}=0
$$

(2) Increase $k$. Find the column-vector of $\Theta$ that provides highest correlation with the residual

$$
c_{k}=\arg \max \left|\left\langle r_{k-1}, \Theta_{n}\right\rangle\right|
$$

(3) Add column $c_{k}$ of $\Theta$ to $\Theta^{\dagger}$ and zero $c_{k}$ from $\Theta$

(4) Solve

and update residual with $\Theta$

$$
\widehat{\sigma}=\arg \min _{\sigma}\|\sigma\|_{2} \quad \text { s.t. } y=\Theta^{\dagger} \sigma
$$

$$
r_{k}=y-\Theta \widehat{\sigma}
$$

(5) Go to step 2 until $k$ reaches stop condition $(k=K)$

Algorithm 1: OMP algorithm description. Adapted from [26].

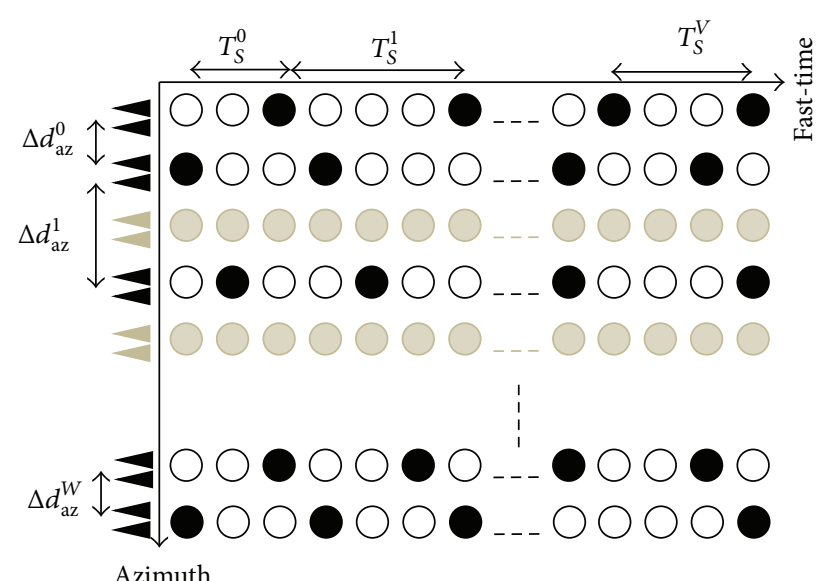

FIGURE 7: Sample selection scheme on full SAR data simulating a real CS sensor. Fast-time samples in white and azimuth measurements in grey are skipped.

For the application proposed in this study, only the scene reflectivity coefficients in $\sigma$ are needed and not the original measurement vector $x$. The recovery problem to be solved can then be formulated as

$$
\widehat{\sigma}=\arg \min _{\sigma}\|\sigma\|_{0} \quad \text { s.t. } y=\Theta \sigma,
$$

being $\|\sigma\|_{0}$ the $l_{0}$-norm of vector $\sigma$. However, the $l_{0}$-norm is an NP-hard problem and solutions may not exist. Since matrix $\Theta$ sufficiently satisfies the RIP condition, the $l_{1}$-norm can be used as a viable alternative to noise-free convex optimization problems

$$
\widehat{\sigma}=\arg \min _{\sigma}\|\sigma\|_{1} \quad \text { s.t. } y=\Theta \sigma .
$$

Optimization of noisy measurements must be approximated since a perfect reconstruction will not be possible [22]. A tolerance threshold $\varepsilon$ related to the data noise level or accepted reconstruction error must be imposed as follows:

$$
\widehat{\sigma}=\arg \min _{\sigma}\|\sigma\|_{1} \quad \text { s.t. }\|\Theta \sigma-y\|_{2}<\varepsilon .
$$

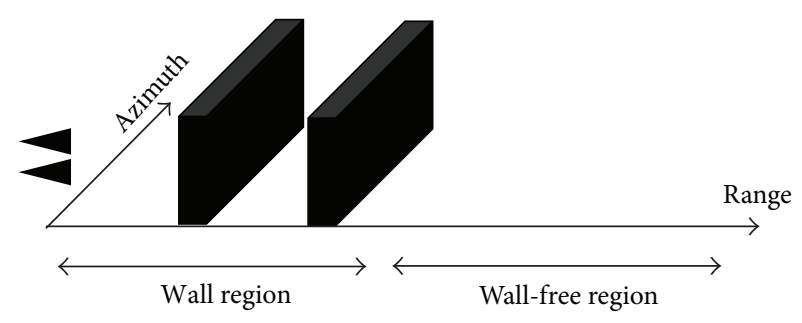

FIGURE 8: Two regions are described and will be treated differently. Walls may appear closer to the sensor. Further away only point targets are expected.

Several categories different from convex optimization or basis pursuit can be found, each one offering multiple algorithms and variations. One of the most popular in terms of simplicity and efficiency is Orthogonal Matching Pursuit (OMP) [25] and its variations [26]. Being a greedy and simple algorithm (see Algorithm 1), OMP requires a prior knowledge or estimation of the sparsity in order to recover a sparse vector. Assessing the sparsity of a scene is not always possible especially in TWI. A relatively performing solution is implementing a stop condition to avoid the algorithm iterating more than what required by the scene. $l_{1}$-norm and OPM will be used in Section 4 .

3.3. CS Random Sampling and Dictionary Structure. Random downsampling in the fast-time and azimuth dimensions implies a substantial reduction in data collection, in measurement time (less azimuth scans), and sensor complexity (fast-time sampling is less demanding). Random sampling schemes must be cautiously imitated when constructing the CS dictionary $\Theta$ with the known signal model. Figure 7 describes how azimuth and fast-time random samplings are generated using the conventional SAR approach as a reference. Sampling intervals $T_{S}^{v}$ and azimuth steps $\Delta d_{a z}^{w}$ follow adjustable random statistical distributions. 


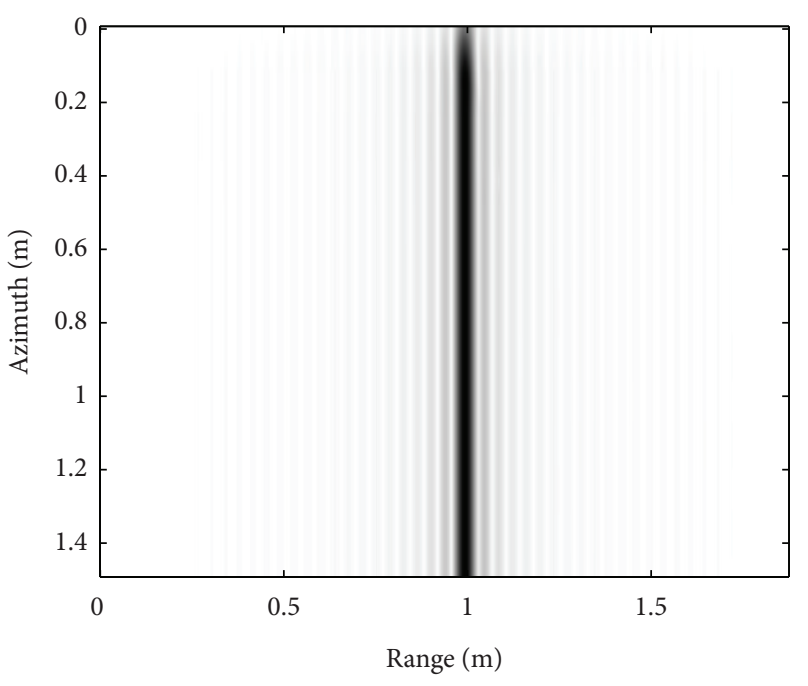

(a)

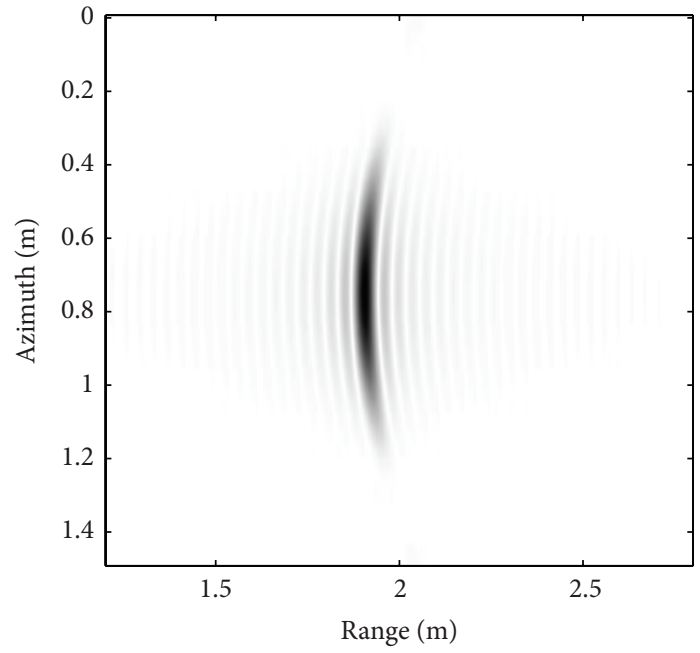

(b)

FIGURE 9: Range-compressed images of a simulated stepped frequency SAR system. (a) a scene containing scatterers modeling a simple wall model located at $0.99 \mathrm{~m}$ in range, used to populate the wall dictionary. (b) a scene containing one-single point target located at range $1.9 \mathrm{~m}$ and azimuth $0.75 \mathrm{~m}$, used to populate the point-target dictionary.

3.4. Modeling CS Dictionaries. CS dictionaries can be modeled according to the type of scene that must be reconstructed. A possible solution for the problem here addressed is considering two regions in the measurement (Figure 8), which also add complexity scalability to CS on TWI scenes as follows.

(i) A region closer to the sensor (wall region) where only walls parallel to the sensors' motion path are expected to appear. Since walls present very strong azimuth redundancies in SAR measurements, the wall region can be reconstructed forcing coarse azimuth and range resolutions in the final imagery. For every possible range in the scene, the wall dictionary will contain in its columns simulated received SAR data based on a simple infinite wall model (Figure 9(a)).

(ii) A second region is defined further away from the sensor, where scatterers other than walls are expected. This region is known as the wall-free region. Sufficient azimuth information is necessary to characterize point targets in the scene, and reducing the number of azimuth positions is not a sound option. For each position of a point target in the scene, received SAR signals are simulated and included in the columns of the point-target dictionary (Figure 9(b)).

3.5. Random Sampling Limitations. Estimating the number of required random samples for CS reconstruction is a complex task since scene sparsity cannot be easily assessed prior to the measurement. However, an approximate expertise can be gained by systematically testing out several random downsampling possibilities. As discussed in Section 3.3, the main restriction will be established by the wall-dictionary reconstruction. Figure 10 shows Peak Signal-to-Noise Ratio (PSNR) reconstruction values, in which from $10 \%$ to $90 \%$

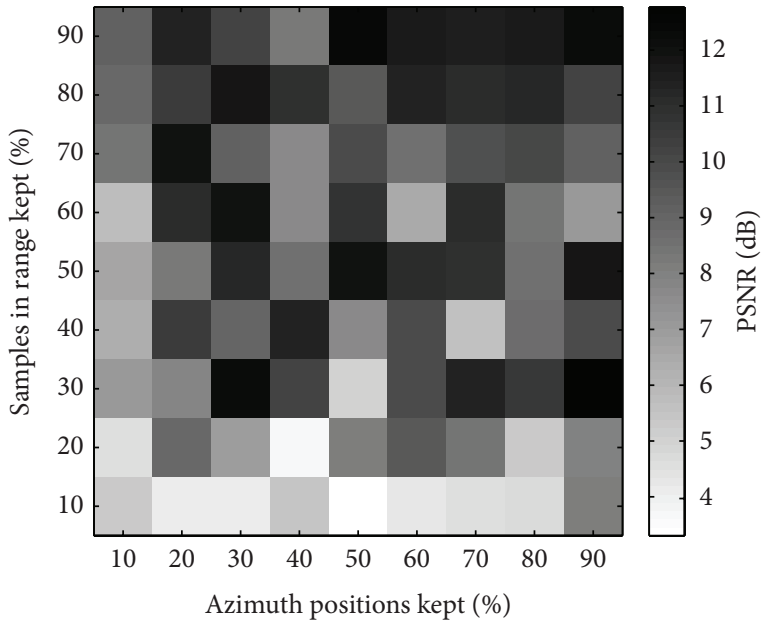

FIGURE 10: PSNR values in $\mathrm{dB}$ for different random downsampling possibilities in fast time and azimuth. A time-domain signal model was used to create the dictionary.

of the azimuth and fast-time samples of a complete SAR TWI measurement were randomly selected. The underlying purpose of the experiment is determining how accurate the image of a wall can be reconstructed if low, medium, or severe downsampling happens and selecting an adequate working point. Analysis of results suggests, as expected, that using severe random downsampling in any dimension impedes an accurate data reconstruction. Using higher values produces an evident improvement in the reconstructions, but those would not yield hardware or computational time reductions. Based on the compromise between accuracy and simplicity, random sampling should range between $20 \%$ and $50 \%$ in the fast time and between $20 \%$ and $50 \%$ for azimuth. 


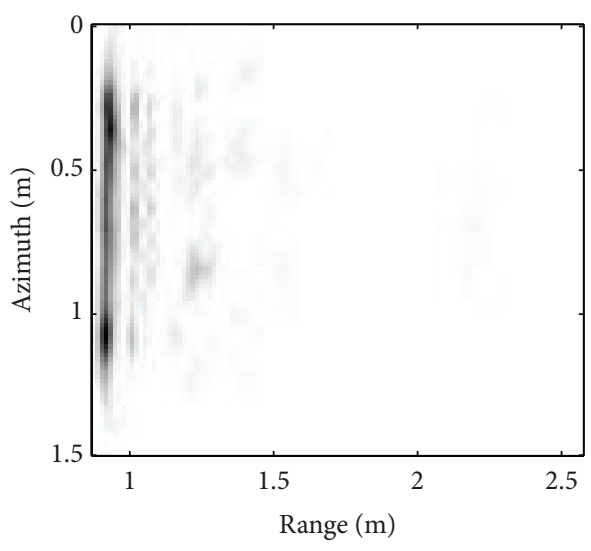

(a)

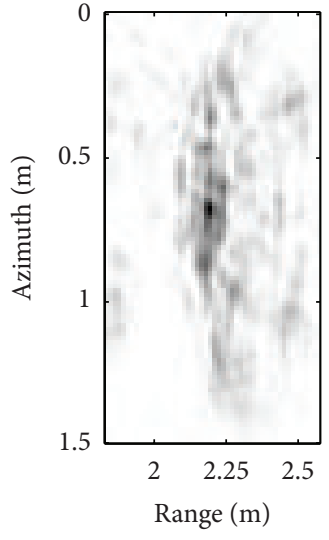

(b)

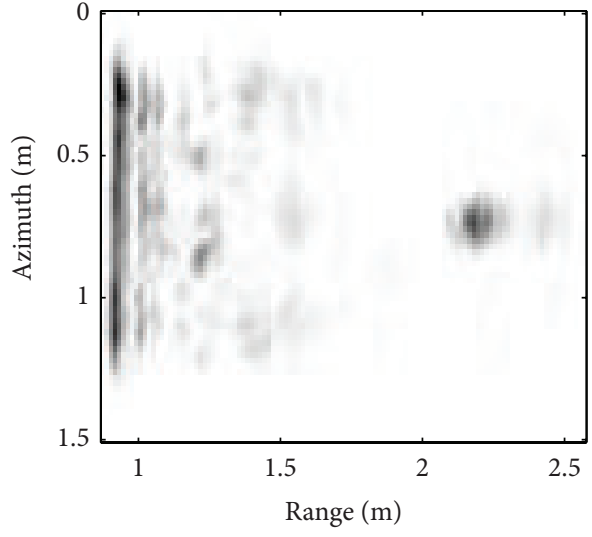

(c)

FIgURE 11: (a) A range-compressed SAR image. The wall is clearly seen. (b) The energy of the corner reflector is spread in azimuth at approximate range $2.25 \mathrm{~m}$. (c) A fully processed SAR image where the presence of a corner reflector is clearly spotted at range $2.25 \mathrm{~m}$.

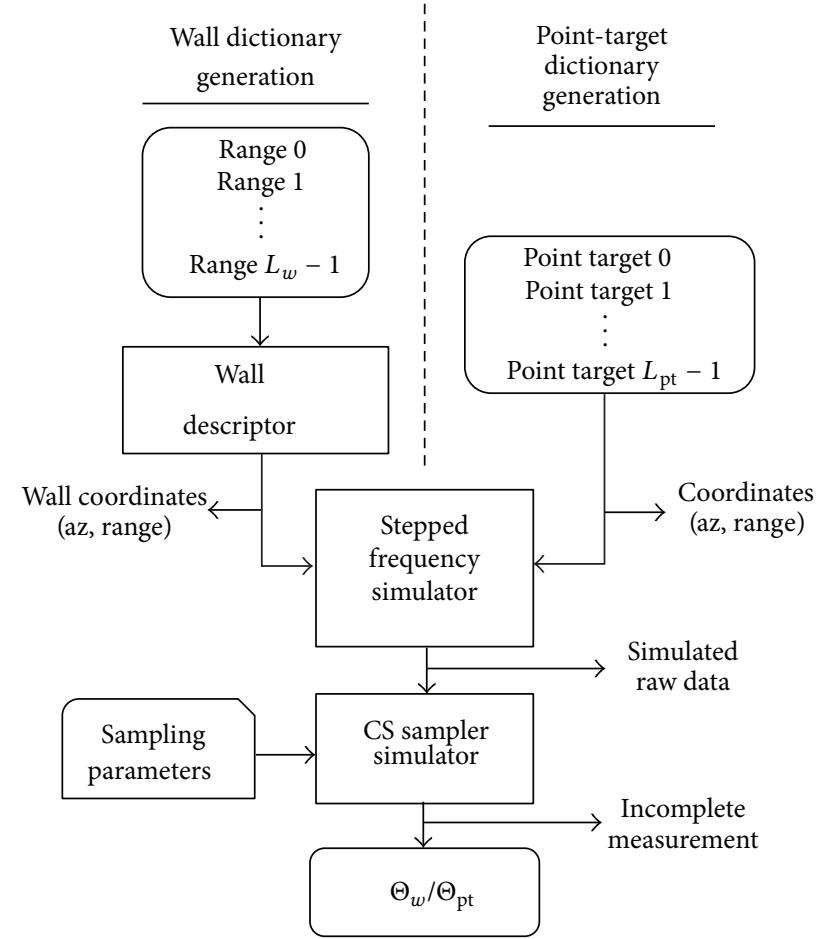

(a)

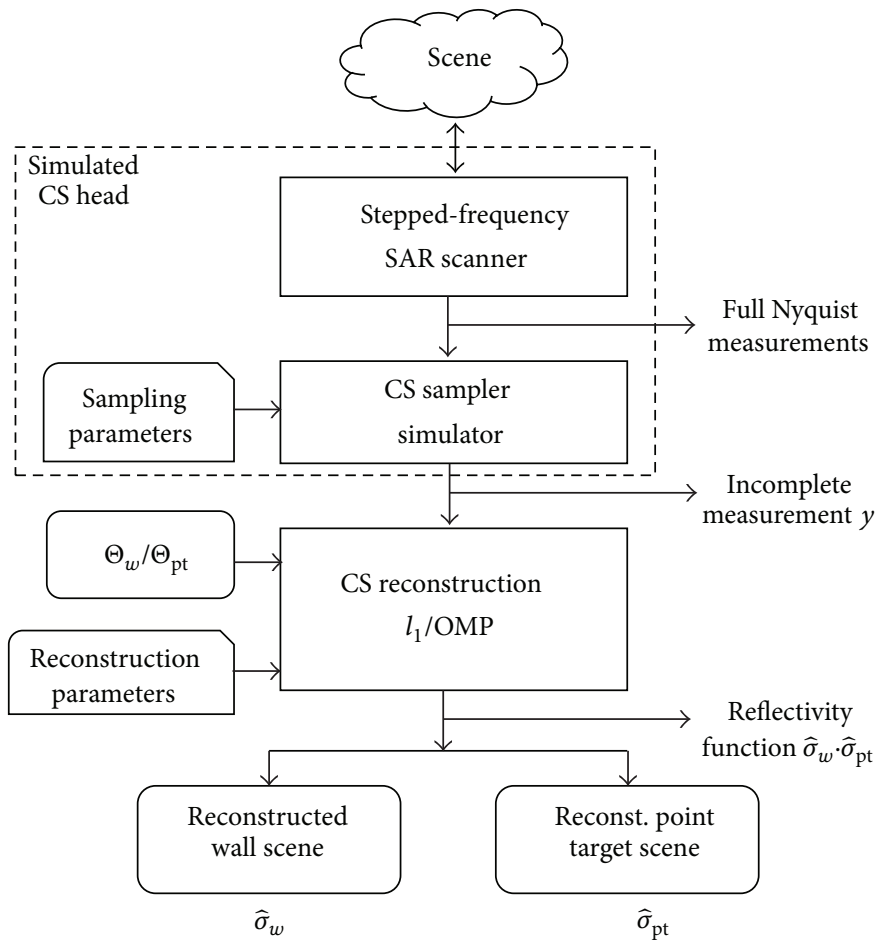

(b)

FIGURE 12: Block diagrams of the (a) wall and point-target dictionary generation and (b) scene reconstruction from actual incomplete measurements.

Given the presented results, this study will use a random downsampling of $50 \%$ of the usual samples for both fasttime and azimuth dimensions in the wall-free region, which implies keeping only $25 \%$ of the necessary samples for conventional SAR processing. Additionally, decimation by two of the azimuth measurements can be safely applied for the wall region, yielding $12.5 \%$ of the original data.
3.6. Practical Experimental Details. The experimental TWI setup uses a conventional SAR system, and original CS measurements cannot be obtained. A full SAR measurement is performed with the presented SF SAR system in order to obtain sufficient fast-time and azimuth information for further random downsampling. The SAR simulator used to model the expected scatterers operates likewise. 


\section{Results and Discussion}

4.1. Conventional SAR TWI Results. The experimental setup depicted in Figures 1 and 5 was measured using the sensor described in Table 1, and conventional time-domain SAR processing was applied. In Figure 11(a), in a rangecompressed image of the acquired SAR signals, the wall is clearly visible in the leftmost side due to the strong reflections generated. In the center image, the received energy from corner reflector behind the wall is greatly spread in azimuth following the hyperbolic structure depicted in Figure 6.

After having taken into account azimuth information, the fully compressed SAR image is reconstructed as shown in Figure 11(c). Indeed, the wall is clearly seen as well as several artifacts in adjacent ranges further from the sensor. Such artifacts correspond to low returns from the second layer of the hollow concrete blocks as well as multiple internal reflections which appear in object-free ranges, as predicted in Section 2.1.

4.2. CS Experimental Results. A CS head and processor are imitated in software following the considerations explained in Sections 2 and 3. Raw SAR data shown in Section 4.1 are sampled at different random sampling rates and reconstructed assuming scene sparsity. In this section, presented results were obtained according to the methodology described in Section 3.4 for efficient wall reconstruction and ranging and behind-the-wall accurate reconstruction. The reconstruction dictionaries are built up using simple wall and point-target models and a stepped-frequency SAR simulator.

Figure 12(a) describes how the signal simulator is fed with coordinates produced by the models, which follows to a CS sampler simulator which downsamples the simulated data in range and azimuth according to the provided sampling parameters. The resulting downsampled data are vectorized and stored in the columns of the wall or point-target dictionary, $\boldsymbol{\Theta}_{w}$ or $\boldsymbol{\Theta}_{\mathrm{pt}}$, respectively. Scene reconstruction of actual measurements is depicted in Figure 12(b). A CS head is partially simulated in software, downsampling the measured signals following a given random sampling scheme identical to that used during the dictionary generation. The CS reconstruction block is fed with the incomplete measurements and the previously generated dictionaries and operational parameters for reconstruction, which may vary depending on the reconstruction algorithms (OMP or the $l_{1}$ norm).

Figure 13 shows the reconstruction of the wall reflectivity coefficients vector $\sigma_{w}$ using OMP and a wall-only dictionary coarsely modeled for efficient processing purposes. The range of maximum reflectivity, $0.95 \mathrm{~m}$, corresponds to the imaged wall, which satisfies expected results.

A more accurate CS dictionary is then used for pointtarget reflectivity coefficients reconstruction, or vector $\sigma_{\mathrm{pt}}$. Based on $\sigma_{w}$, it is assumed that one-single wall was present in the scene. A guard range is kept to avoid the clutter region in ranges immediately after the wall (refer to Figures 3, 6 , and 8). Figures 14-16 present reconstructed scenes, where a

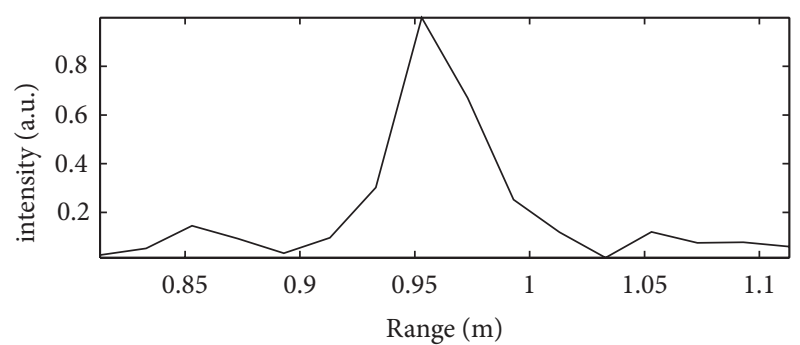

FIGURE 13: CS reconstruction of the scene using a coarse wall dictionary for wall ranging. The actual wall was located at $0.95 \mathrm{~m}$.

corner reflector is expected to appear at range $2.25 \mathrm{~m}$, for several range downsampling options $(60 \%, 50 \%$, and $40 \%)$. OMP generates simplified but correct reconstructions of $\sigma_{\mathrm{pt}}$ two or three orders of magnitude faster, on average, than the $l_{1}$-norm algorithm.

Figure 14 depicts results using downsampling factors of $50 \%$ and $60 \%$ for azimuth and fast time, respectively. Accurate ranging of the corner reflector is obtained $(2.25 \mathrm{~m})$ and comparable to that of the conventional SAR imagery shown in Figure 10 right. Results generated by the $l_{1}$-norm clearly indicate where the corner reflector is located, while OPM extracts the most significant reflectivity coefficients. Given the theoretical range resolution of $37.5 \mathrm{~mm}$, the two targets extracted using OMP can be assumed to be in the same resolution cell. As the number of fast-time samples kept is reduced (Figures 15 and 16), inaccuracies in the corner reflector's positioning and false alarms appear, although a general agreement can be reached on the presence of a strong reflector in the scene.

\section{Conclusions and Future Work}

In this work, CS has been successfully applied to TWI of a real concrete wall. SAR measurements were preprocessed in software to simulate a CS sensor which would perform random sampling in the fast-time and azimuth dimensions. The particular considerations regarding the application of CS onto SAR measurements for TWI were addressed. By using CS, any kind of SAR processing was avoided. A conceptually simple procedure was designed to automatically distinguish between wall and wall-free regions, which exploits the possibilities that CS can offer and reduces computational complexity and avoids having to treat wall clutter. A systematic investigation was performed to determine the minimum random downsampling with respect to conventional SAR TWI without compromising reconstruction accuracy. Data volume was reduced to as low as $25 \%$ of the usual required samples, enabling measurement time and hardware complexity reduction.

Future work is focused on finding alternatives to further exploit the CS capabilities for TWI. Some of the lines of investigation the authors are following related to signal processing and CS are as follows. 


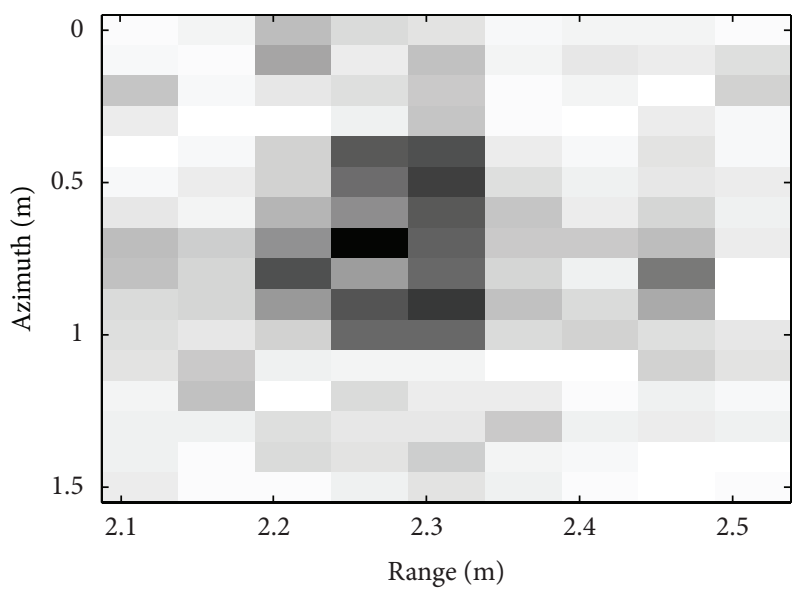

(a)

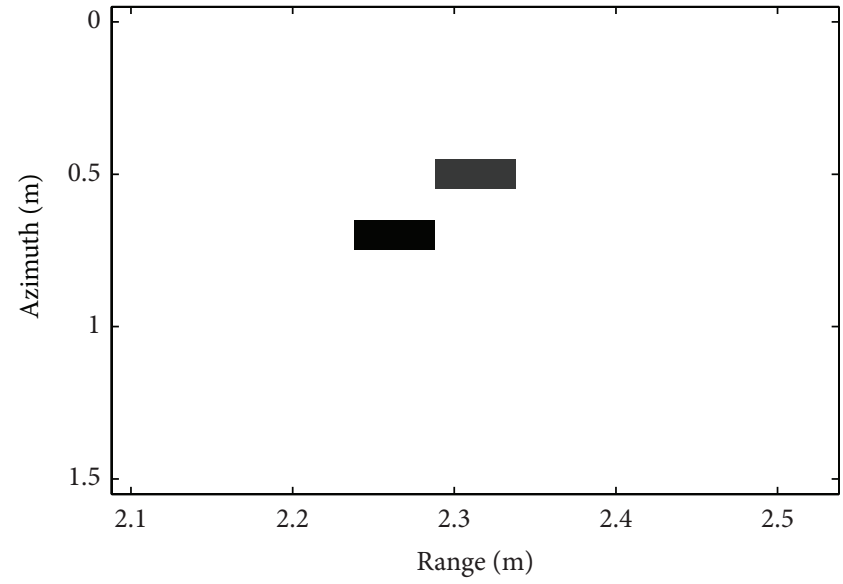

(b)

FIGURE 14: CS reconstructions of the behind-the-wall scene using a point-target dictionary. Downsampling factors were set to $60 \%$ for fasttime and $50 \%$ for azimuth. (a) $l_{1}$-norm; (b) OMP.

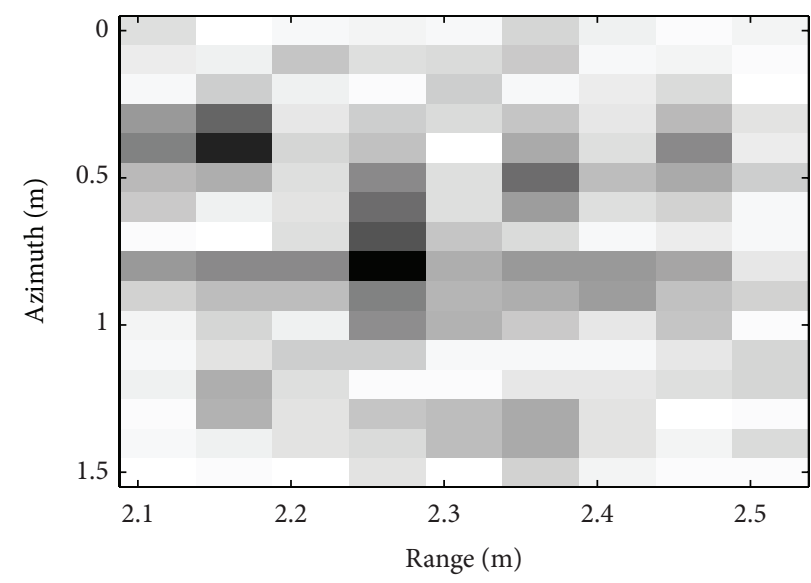

(a)

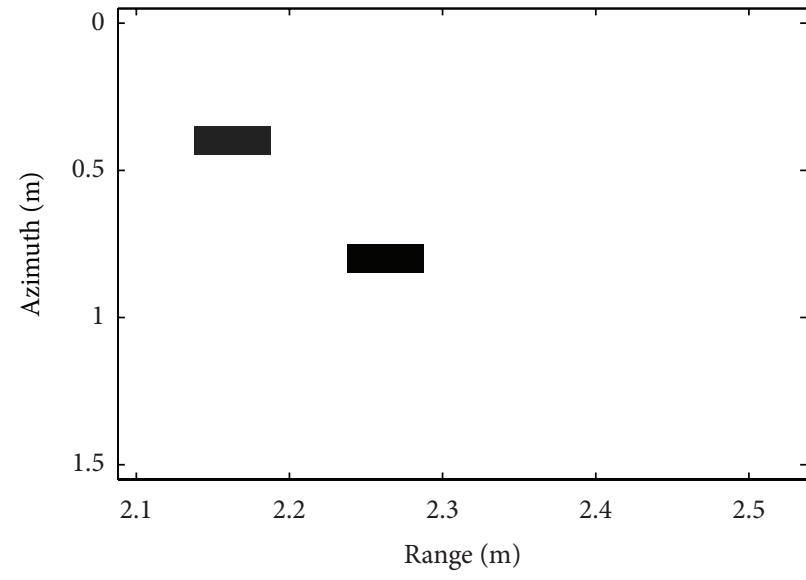

(b)

FIGURE 15: CS reconstructions of the behind-the-wall scene using a point-target dictionary. Downsampling factors were set to $50 \%$ for fasttime and $50 \%$ for azimuth. (a) $l_{1}$-norm; (b) OMP.

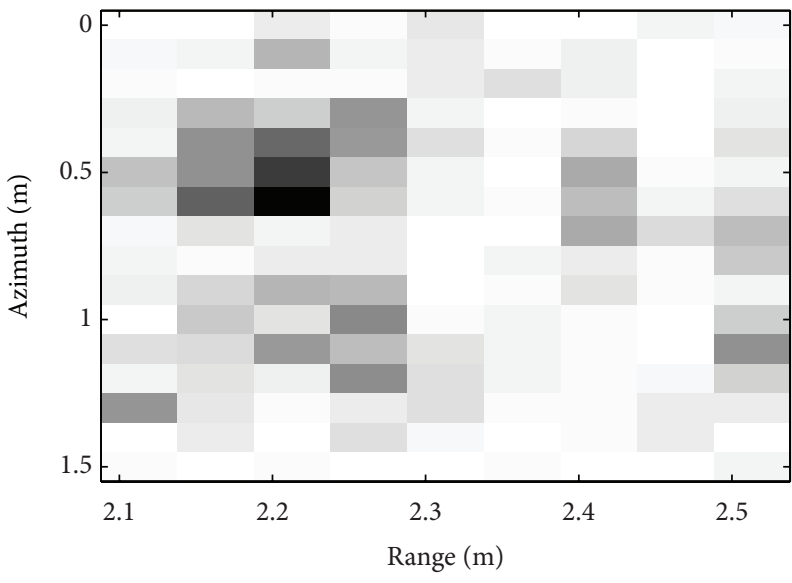

(a)

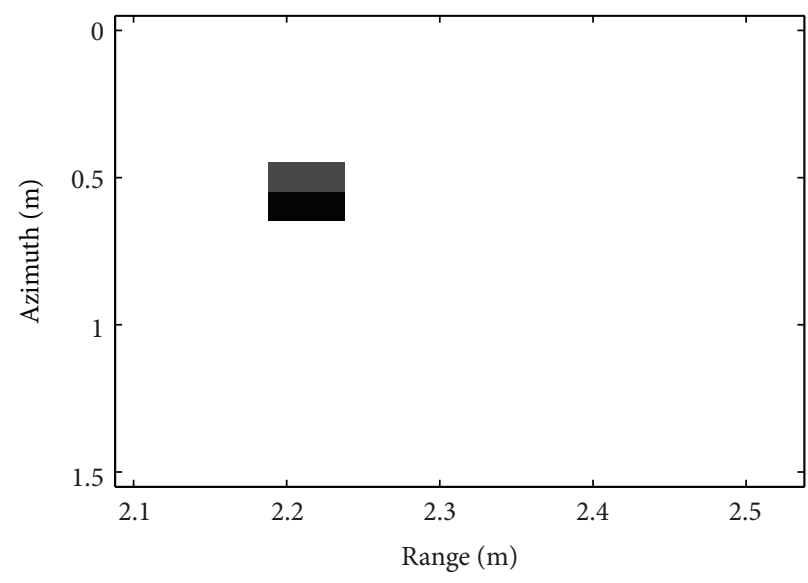

(b)

FIGURE 16: CS reconstructions of the behind-the-wall scene using a point-target dictionary. Downsampling factors were set to $40 \%$ for fasttime and $50 \%$ for azimuth. (a) $l_{1}$-norm; (b) OMP. 
(i) Reducing the effects of strong reflections from the wall introduces the reconstruction of point-target regions and improves the accuracy of detection. The authors currently are working on strategies to overcome these negative effects.

(ii) Multiple echoes and patterns due to the wall structure appear in further adjacent ranges. Using a complete SAR model of a wall will help identify which scatterers can be considered as wall clutter or targets of interest behind the wall.

(iii) Range inaccuracies due to inhomogeneous transmission media will be corrected to provide a correct range estimation. A complete wall model will be developed based on existing publications.

(iv) The SF system used in the experiments as well as the antennas has a frequency-dependent performance. A complete SAR signal model must be developed to create CS dictionaries as accurate as possible. Moreover, the mutual interaction between both adjacent antennas modifies their radiation patterns. The antennas will be measured in an anechoic chamber to include this effect in the complete signal model.

(v) CS applied to 3D SAR imaging proposes challenging open questions regarding sparsity assessment and strategies to guarantee the viability the of proposed methodologies in this work.

(vi) Data transformation bases will be studied to enhance data sparsity and facilitate accurate scene reconstructions with even less random samples and simpler hardware.

(vii) Other efficient sparse data reconstruction algorithms will be explored and modified to the TWI needs where applicable.

\section{Acknowledgments}

The authors of this study would like to show their gratitude to the OMRA and LEMA laboratories of the Royal Military Academy, Belgium, for providing both technical support and logistic support during construction and measurement of the presented experimental scenes.

\section{References}

[1] O. Landron, M. J. Feuerstein, and T. S. Rappaport, "A comparison of theoretical and empirical reflection coefficients for typical exterior wall surfaces in a mobile radio environment," IEEE Transactions on Antennas and Propagation, vol. 44, no. 3, pp. 341-351, 1996.

[2] A. Muqaibel, A. Safaai-Jazi, A. Bayram, A. M. Attiya, and S. M. Riad, "Ultra-wideband through-the-wall propagation," IEE Proceedings, pp. 581-588, 2005.

[3] T. S. Ralston, G. L. Charvat, and J. E. Peabody, "Real-time through-wall imaging using an ultrawideband multiple-input multiple-output (MIMO) phased array radar system," in Proceedings of the 4th IEEE International Symposium on Phased Array Systems and Technology (Array '10), pp. 551-558, October 2010.
[4] F. Ahmad, M. G. Amin, and P. Setlur, "Through-the-wall target localization using dual-frequency CW radars," in Proceedings of the Sensors, and Command, Control, Communications, and Intelligence (C3I) Technologies for Homeland Security and Homeland Defense V, vol. 6201 of Proceedings of SPIE, 62010H, May 2006.

[5] Y. Yoon and M. G. Amin, "Spatial filtering for wall-clutter mitigation in through-the-wall radar imaging," IEEE Transactions on Geoscience and Remote Sensing, vol. 47, no. 9, pp. 3192-3208, 2009.

[6] R. Solimene, F. Soldovieri, G. Prisco, and R. Pierri, "Threedimensional through-wall imaging under ambiguous wall parameters," IEEE Transactions on Geoscience and Remote Sensing, vol. 47, no. 5, pp. 1310-1317, 2009.

[7] W. Zhang, L. Li, and F. Li, "Autofocusing imaging through the unknown building walls," in Proceedings of the Asia Pacific Microwave Conference (APMC '08), pp. 1-5, December 2008.

[8] D. K. P. Tan, M. Lesturgie, H. Sun, W. Li, and Y. Lu, "Gsm based through-the-wall passive radar demonstrator for motion sensing," in Proceedings of the New Trends for Environmental Monitoring Using Passive Systems (PASSIVE '08), pp. 1-4, Hyères, France, October 2008.

[9] K. Chetty, G. E. Smith, and K. Woodbridge, "Through-thewall sensing of personnel using passive bistatic wifi radar at standoff distances," IEEE Transactions on Geoscience and Remote Sensing, vol. 50, no. 4, pp. 1218-1226, 2012.

[10] I. G. Cumming and F. H. Wong, Digital Processing of Synthetic Aperture Radar Data: Algorithms and Implementation, Artech House, Norwood, Mass, USA, 2005.

[11] M. Leigsnering, C. Debes, and A. M. Zoubir, "Compressive sensing in through-the-wall radar imaging," in Proceedings of the 36th IEEE International Conference on Acoustics, Speech, and Signal Processing (ICASSP '11), pp. 4008-4011, May 2011.

[12] F. Ahmad and M. G. Amin, "Partially sparse reconstruction of behind-the-wall scenes," in Proceedings of the SPIE Symposium on Defense, Security, and Sensing, Compressive Sensing Conference, Baltimore, Md, USA, April 2012.

[13] Q. Huang, L. Qu, B. Wu, and G. Fang, "UWB through-wall imaging based on compressive sensing," IEEE Transactions on Geoscience and Remote Sensing, vol. 48, no. 3, pp. 1408-1415, 2010.

[14] E. Lagunas, M. G. Amin, F. Ahmad, and M. Najar, "Compressive sensing for through wall radar imaging of stationary scenes using arbitrary data measurements," in Proceedings of the 11th International Conference on Information Science, Signal Processing and their Applications (ISSPA '12), pp. 1347-1352, July 2012.

[15] D. L. Donoho, "Compressed sensing," IEEE Transactions on Information Theory, vol. 52, no. 4, pp. 1289-1306, 2006.

[16] (n. d.), Schwarzbeck Mess-Elektronik, Double Ridged Broadband Horn BBHA, 9120 D, http://www.schwarzbeck.de/ Datenblatt/k9120d.pdf.

[17] C. Thajudeen, A. Hoorfar, F. Ahmad, and T. Dogaru, "Measured complex permittivity of walls with different hydration levels and the effect on power estimation of twri target returns," Progress in Electromagnetics Research B, vol. 30, pp. 177-199, 2011.

[18] R. Bamler, "A comparison of range-Doppler and wavenumber domain SAR focusing algorithms," IEEE Transactions on Geoscience and Remote Sensing, vol. 30, no. 4, pp. 706-713, 1992.

[19] I. G. Cumming, Y. L. Neo, and F. H. Wong, "Interpretations of the omega-K algorithm and comparisons with other algorithms," in Proceedings of the IEEE International Geoscience and 
Remote Sensing Symposium (IGARSS '03), pp. 1455-1458, July 2003.

[20] H. L. Van Trees, "Detection of signals-estimation of signal parameters," in Detection, Estimation, and Modulation Theory: Detection, Estimation, and Linear Modulation Theory, John Wiley \& Sons, New York, NY, USA, 2002.

[21] C. E. Shannon, "A mathematical theory of communication," The Bell Systems Technical Journal, vol. 27, pp. 379-423, 623-656, 1948.

[22] E. J. Candès, J. K. Romberg, and T. Tao, "Stable signal recovery from incomplete and inaccurate measurements," Communications on Pure and Applied Mathematics, vol. 59, no. 8, pp. 12071223, 2006.

[23] E. J. Candès, J. Romberg, and T. Tao, "Robust uncertainty principles: exact signal reconstruction from highly incomplete frequency information," IEEE Transactions on Information Theory, vol. 52, no. 2, pp. 489-509, 2006.

[24] H. Rauhut, "Compressive sensing and structured random matrices," in Theoretical Foundations and Numerical Methods for Sparse Recovery, M. Fornasier, Ed., vol. 9 of Radon Series on Computational and Applied Mathematics, pp. 1-92, deGruyter, Berlin, Germany, 2010.

[25] T. Blumensath and M. E. Davies, "In greedy pursuit of new directions: (nearly) orthogonal matching pursuit by directional optimisation," in Proceedings of the 15th European Signal Processing Conference (EUSIPCO '07), Poznan, Poland, September 2007.

[26] J. A. Tropp and D. Needell, "CoSaMP: iterative signal recovery from incomplete and inaccurate samples," presented at CoRR, 2008. 

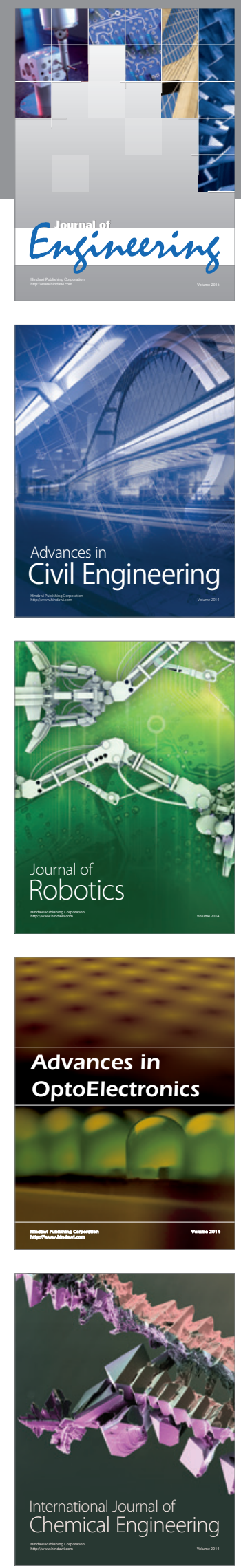

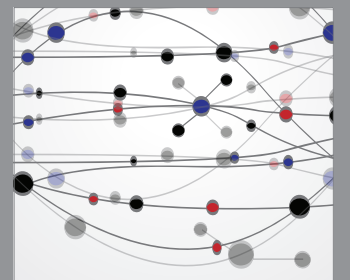

The Scientific World Journal
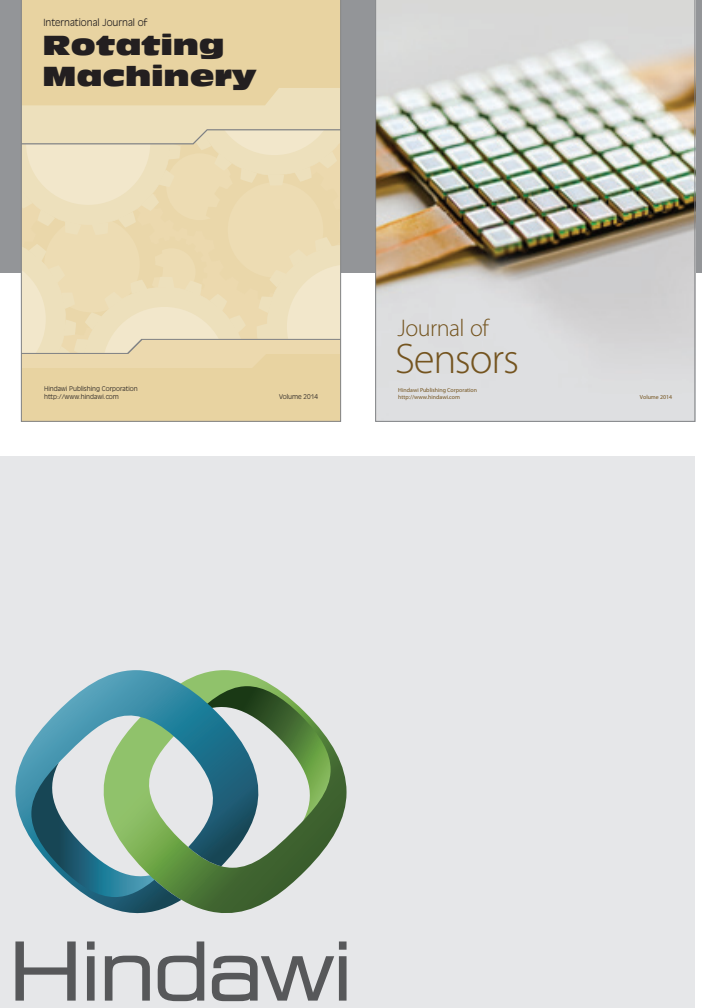

Submit your manuscripts at http://www.hindawi.com
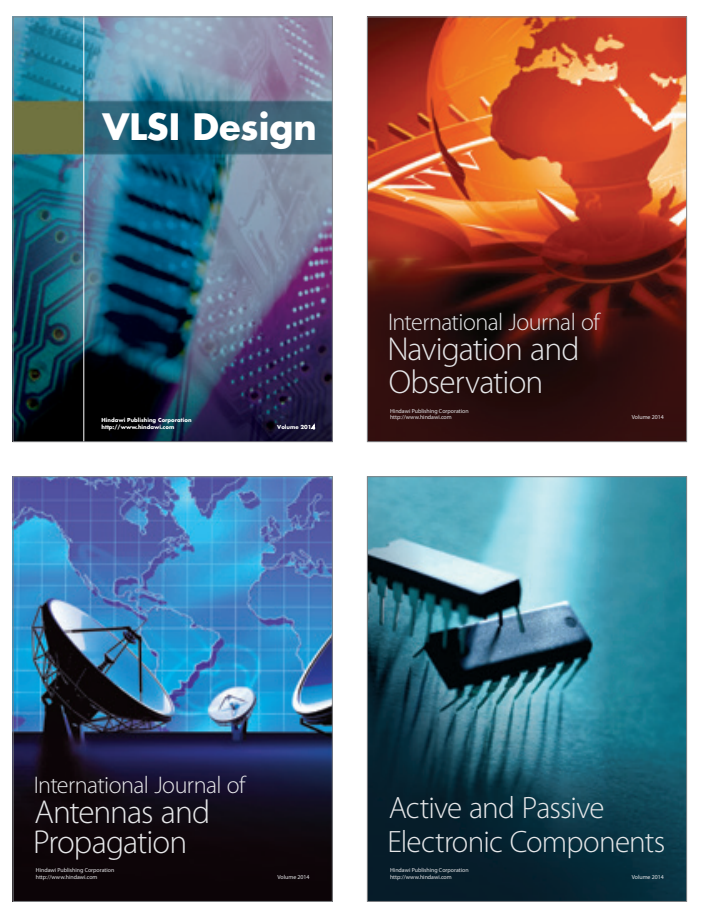
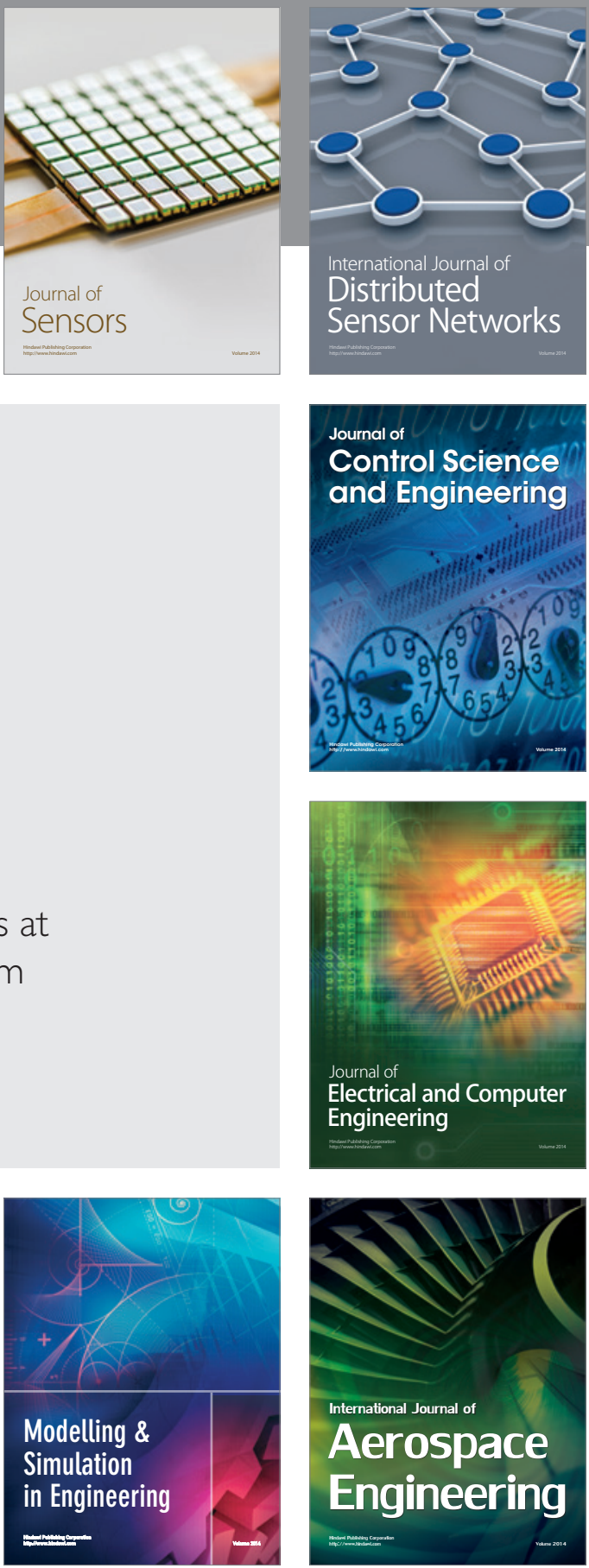

Journal of

Control Science

and Engineering
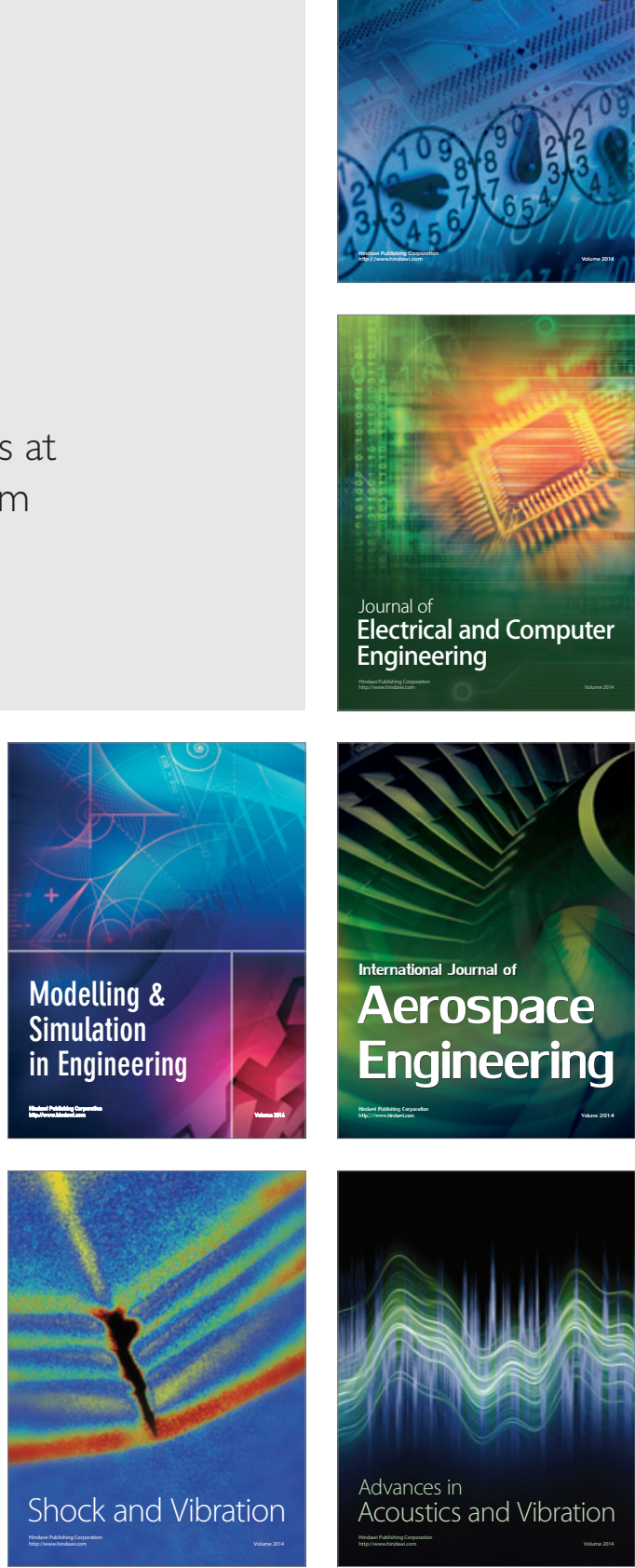